\title{
Determining the cosmic equation of state using future gravitational wave detectors
}

\author{
Z.-H. Zhu ${ }^{1,2}$, M.-K. Fujimoto ${ }^{1}$, and D. Tatsumi ${ }^{1}$ \\ 1 National Astronomical Observatory, 2-21-1, Osawa, Mitaka, Tokyo 181-8588, Japan \\ ${ }^{2}$ National Astronomical Observatories, Chinese Academy of Sciences, Beijing 100012, PR China \\ Received 13 June 2000 / Accepted 22 March 2001
}

\begin{abstract}
The expected chirp mass distribution of observed events for future gravitational wave detectors is extensively investigated in the presence of an exotic fluid component with an arbitrary equation of state, $-1 \leq$ $\omega_{x} \equiv p_{x} / \rho_{x}<0$, i.e., the so-called dark energy component. The results for a flat model dominated by dark energy are compared to those for the standard flat model dominated by cold dark matter. It is found that for a flat universe the chirp mass distribution shows a sensitive dependence on $\omega_{x}$, which may provide an independent and robust constraint on the cosmic equation of state.
\end{abstract}

Key words. cosmology: theory - gravitational waves

\section{Introduction}

Upcoming interferometric detectors of gravitational waves such as LIGO (Abramovici et al. 1992), VIRGO (Caron et al. 1997), GEO (Danzmann et al. 1997) and TAMA (Kuroda 1997) will produce a flood of data when they come online in a few years. Advanced detectors in LIGO/VIRGO and LCGT (Kuroda et al. 1999) will even be able to see neutron star binaries (NS/NS) out to cosmological redshifts. Because the accuracy in the measurement of the signal strength can be better than $10 \%$, and the accuracy in the measurement of the chirp mass (which characterizes the frequency sweep of a binary inspiral) can be better than 0.1\% (Finn \& Chernoff 1993; Cutler \& Flanagan 1994), observations of NS/NS inspiral events can be used to determine the universe's Hubble constant, deceleration parameter and cosmological constant (Schutz 1986, 1989; Marković 1993; Chernoff \& Finn 1993; Finn 1996; Wang \& Turner 1997). In particular, Finn (1996) and Wang \& Turner (1997) explicitly pointed out that the chirp mass spectrum of observed neutron star binary inspiral events can be quite sensitive to cosmological constant.

In this paper, we investigate the possibility of using the future gravitational wave data to constrain dark energy, an exotic fluid component in the universe with an arbitrary equation of state, $-1 \leq \omega_{x} \equiv p_{x} / \rho_{x}<0$, whose existence has been well motivated by various astronomical observations and theoretical arguments (refer to a recent review, Turner 1999). While the cosmological constant (vacuum energy) can apparently succeed in explaining the accelerating expansion of the universe which has stirred cos-

Send offprint requests to: Z.-H. Zhu, e-mail: zong-hong.zhu@nao.ac.jp mologists after observations and measurements of distant Ia type supernovae (SNeIa) (Perlmutter et al. 1999; Riess et al. 1998), it suffers from the difficulty in understanding of the observed value in the framework of modern quantum field theory (Weinberg 1989; Carroll et al. 1992) and the "coincidence problem", the issue of explaining the initial conditions necessary to yield the near-coincidence of the densities of matter and the cosmological constant component today. In this case, a dark energy component with generally negative pressure has been invoked. Examples of dark energy include the evolving scalar field (Caldwell et al. 1998; Zlatev et al. 1999; Steinhardt et al. 1999), the smooth noninteracting component (Turner \& White 1997; Chiba et al. 1997) and the frustrated network of topological defects in which $\omega_{x}=-\frac{n}{3}$, with $n$ being the dimension of the defect (Spergel \& Pen 1997). Some observational constraints for these models have been extensively analyzed in the literature, including the redshift-luminosity distance relation based on distant SNeIa (Garnavich et al. 1998), gravitational lensing statistics (Cooray \& Huterer 1999; Zhu \& Cao 1999; Zhu 2000), Einstein ring system (Futamase \& Hamana 1999), the anisotropy of the Cosmic Microwave Background Radiation (CMBR) (Huey et al. 1999), the angular size-redshift relation of distant extragalatic sources (Lima \& Alcaniz 2000a), the age of highredshift galaxies (Lima \& Alcaniz 2000b), and the combinations of SNeIa measurements with either gravitational lensing (Waga \& Miceli 1999) or CMBR (Efstathiou 1999) or large-scale structure (Perlmutter et al. 1999) or the time variations of the redshift of quasars (Nakamura \& Chiba 1999). A combined maximum likelihood analysis has suggested a range of equation-of-state for quintessence, $-1 \leq$ $w_{x} \lesssim-0.6$ (see Wang et al. 2000 for an elegant summary). 
Because the determination of the amount and nature of dark energy is emerging as one of the most important challenges in cosmology, it is worthwhile to investigate other observational aspects of its equation of state. In this work, from calculating the expected chirp mass spectrum of observed NS/NS inspiral events for future gravitational wave detectors in the presence of a dark energy component, we find a sensitive dependence of chirp mass distribution on $\omega_{x}$ for a flat universe, which could provide an independent and robust constraint on the cosmic equation of state. For calculations of this work, our notation and approach mainly follow those described by Finn (1996).

\section{The chirp mass spectrum of NS/NS inspiral events}

The NS/NS inspirals are the targets for future gravitational wave detectors, whose merger rate at redshift $z$ per unit observer time interval per unit physical volume is, $\dot{n}=\dot{n}_{0} F(z)(1+z)^{2}$, where $F(z)$ describes the NS/NS merge rate evolution, and $\dot{n}_{0}\left(\simeq 10^{-7} h \mathrm{Mpc}^{-3} \mathrm{yr}^{-1}\right)$ is the local NS/NS merger rate per unit volume (Phinney 1991). In the case of no proper evolution $F(z)=1$. The evolution of the NS/NS merger rate has been studied by several authors (e.g., Tutukov \& Yungelson 1993; Lipunov et al. 1995). Because recent observations of field galaxies out to redshift $z \sim 5$ (see e.g. Madau et al. 1998; Steidel et al. 1999) have provided a means of modeling the evolution of the star formation rate (SFR), one can now compute the birth and merger rates of binary systems by taking into account the time-delay. Using population synthesis model, Schneider et al. (2000) have calculated birth and merger rate evolution of various kinds of binaries. Our best fit to their results for NS/NS merger rate from hierarchical scenario (Fig. 8 of Schneider et al. 2000) takes

$$
F(z)=1+11.5 z^{2}-13.6 z^{3}+5.37 z^{4}-0.70 z^{5} \quad z \in(0,3) .(1)
$$

For a NS/NS inspiral source located at redshift $z$, the detectors measure its chirp mass, $\mathcal{M} \equiv \mathcal{M}_{0}(1+z)$ where the intrinsic chirp mass, $\mathcal{M}_{0} \equiv \mu^{3 / 5} M^{2 / 5}$, with $\mu$ and $M$ being the binary's reduced and total mass respectively. Therefore, the chirp mass spectrum represents directly the source redshift distribution if $\mathcal{M}_{0}$ is constant. Because we are considering NS/NS binaries, $\mathcal{M}_{0}=1.19 M_{\odot}$ will be used below, corresponding to a typical neutron star mass $m=1.37 M_{\odot}$. The signal-tonoise ratio $\rho$ of an event observed by a given detector is $\rho=8 \Theta\left(r_{0} / D_{L}\right)\left(\mathcal{M} / 1.2 M_{\odot}\right)^{5 / 6} \zeta\left(f_{\max }\right)$, where $D_{\mathrm{L}}$ is the luminosity distance from the detector to the source. $\zeta(\simeq 1$ for our case) describes the overlap of the signal power with the detector bandwidth, while $r_{0}$ gives an overall sense of the depth to which the detector can reach. The signalto-noise ratio of an inspiraling binary with a single interferometric detector depends on the relative orientation of the source, which is confined to the angular orientation function $\Theta$. From observations of binary inspiral in a single interferometer we can measure $\rho$ and $\mathcal{M}$ but not $\Theta$.
Fortunately, even though $\Theta$ cannot be measured, its probability distribution can be approximated by (Finn 1996)

$$
P_{\Theta}(\Theta)= \begin{cases}5 \Theta(4-\Theta)^{3} / 256 & \text { if } 0<\Theta<4 \\ 0 & \text { otherwise }\end{cases}
$$

which is related to $P_{\rho}\left(\rho \mid \mathcal{M}_{0}, \mathcal{M}\right)$, the probability distribution of signal-to-noise ratio of events with chirp mass $\mathcal{M}$, through

$$
\begin{aligned}
P_{\rho}\left(\rho \mid \mathcal{M}_{0}, \mathcal{M}\right)= & P_{\Theta}\left[\frac{\rho}{8} \frac{D_{L}(Z)}{r_{0}}\left(\frac{1.2 M_{\odot}}{\mathcal{M}}\right)^{5 / 6}\right] \\
& \times \frac{D_{L}(Z)}{8 r_{0}}\left(\frac{1.2 M_{\odot}}{\mathcal{M}}\right)^{5 / 6},
\end{aligned}
$$

where $Z=\mathcal{M} / \mathcal{M}_{0}-1$. Then, in a homogeneous and isotropic universe we get the rate at which binary inspiral signals corresponding to chirp mass $\mathcal{M}$ and $\rho$ greater than $\rho_{0}$ are detected

$$
\begin{aligned}
\frac{\mathrm{d} \dot{N}\left(>\rho_{0}, \mathcal{M}\right)}{\mathrm{d}\left(\mathcal{M} / \mathcal{M}_{0}\right)}= & \left.\dot{n} \cdot 4 \pi D_{\mathrm{A}}^{2} \frac{c \mathrm{~d} t}{\mathrm{~d} z}\right|_{Z} \cdot \int_{\rho_{0}}^{\infty} P_{\rho}\left(\rho \mid \mathcal{M}_{0}, \mathcal{M}\right) \mathrm{d} \rho \\
= & \left.4 \pi \dot{n}_{0} F(Z)\left(\frac{D_{\mathrm{L}}(Z)}{1+Z}\right)^{2} \frac{c \mathrm{~d} t}{\mathrm{~d} z}\right|_{Z} \\
& \times C_{\Theta}\left[\frac{\rho_{0}}{8} \frac{D_{\mathrm{L}}(Z)}{r_{0}}\left(\frac{1.2 M_{\odot}}{\mathcal{M}}\right)^{5 / 6}\right], \quad(4)
\end{aligned}
$$

where $D_{\mathrm{A}}$ is the angular diameter distance from the detector to the source, $C_{\Theta}(x)$ is the probability that a given detector receives a binary inspiral with chirp mass $\mathcal{M}$ and signal-to-noise ratio greater than $\rho_{0}$ :

$C_{\Theta}(x)= \begin{cases}(1+x)(4-x)^{4} / 256, & \text { if } 0<x<4 \\ 0, & \text { otherwise }\end{cases}$

An accumulation of observed NS/NS binary inspiral events will give a chirp mass spectrum

$P\left(\mathcal{M} \mid \rho_{0}\right)=\frac{\mathrm{d} \dot{N}\left(>\rho_{0}, \mathcal{M}\right) / \mathrm{d} \mathcal{M}}{\dot{N}\left(>\rho_{0}\right)}$

\section{Determining the cosmic equation of state}

In order to explicitly show the dependence of the chirp mass spectrum on the cosmic equation of state, one needs to consider it in the context of general FriedmannRobertson-Walker (FRW) cosmologies in the presence of a dark energy component. For general FRW cosmologies, the metric of space time is described by (in the $c=1$ unit):

$\mathrm{d} s^{2}=-\mathrm{d} t^{2}+R^{2}(t)\left[\mathrm{d} \chi^{2}+f^{2}(\chi)\left(\mathrm{d} \theta^{2}+\sin ^{2} \theta \mathrm{d} \phi^{2}\right)\right]$,

where $f(\chi)=\chi$ for a flat universe $(k=0), f(\chi)=\sin \chi$ for a closed universe $(k=+1)$, and $f(\chi)=\sinh \chi$ for an open universe $(k=-1)$. The comoving distance $\chi$ is (Zhu 1998; Zhu \& Cao 1999)

$\chi= \begin{cases}\int_{0}^{z} \frac{\mathrm{d} z}{\sqrt{\sum_{i} \Omega_{i}(1+z)^{3\left(1+\omega_{i}\right)}}} & (k=0) \\ \left|\Omega_{k}\right|^{1 / 2} \int_{0}^{z} \frac{\mathrm{d} z}{\sqrt{\sum_{i} \Omega_{i}(1+z)^{3\left(1+\omega_{i}\right)}+\Omega_{k}(1+z)^{2}}} & (k= \pm 1)\end{cases}$ 

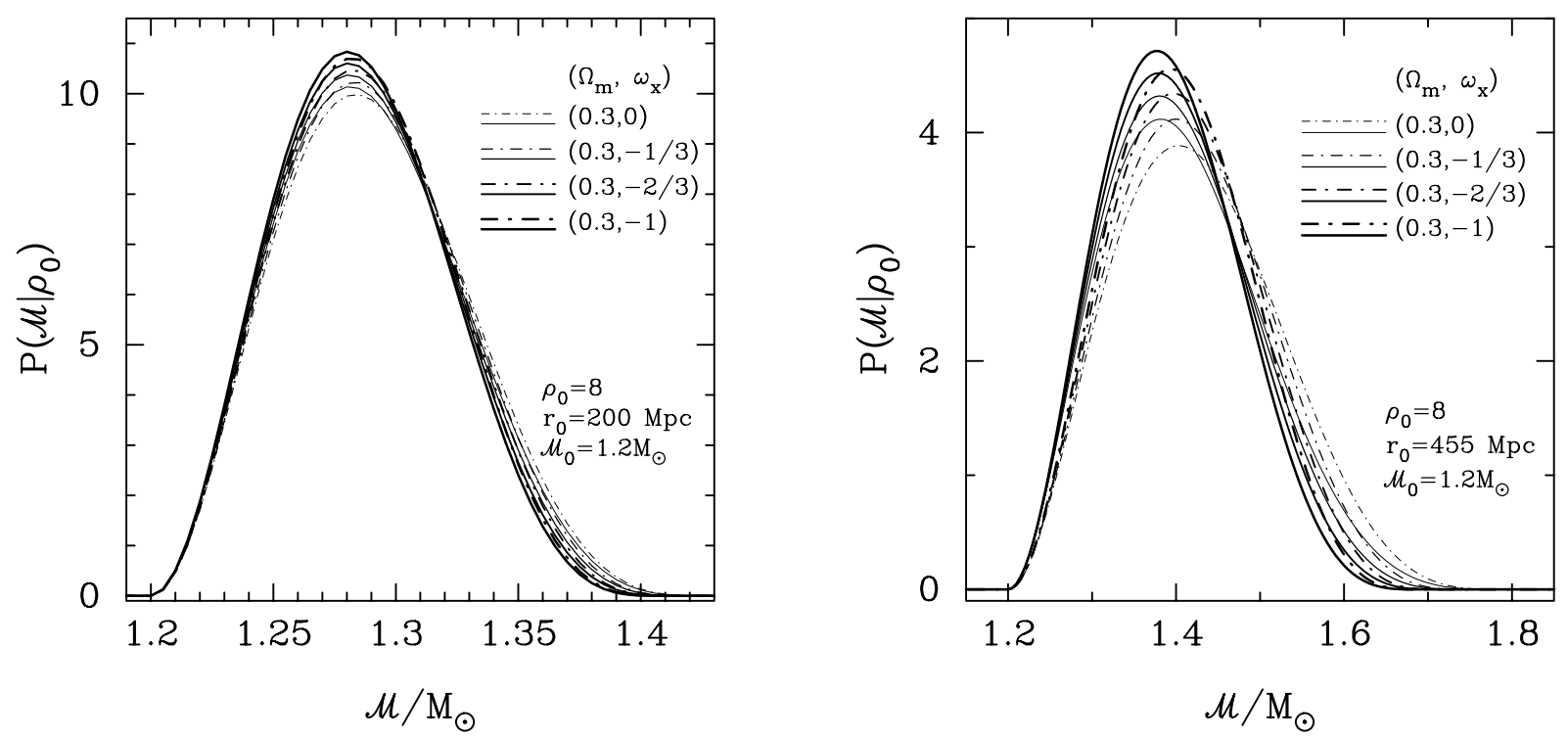

Fig. 1. The chirp mass spectrum of NS/NS binary inspirals for a flat universe, $\left(\Omega_{m}, \Omega_{x}\right)=(0.3,0.7)$, with $\omega_{x}=0$ (SCDM model), $-1 / 3,-2 / 3$ and -1 ( $\Lambda \mathrm{CDM}$ model). The detector depth is assumed to be $r_{0}=200 \mathrm{Mpc}$ (left panel) and $r_{0}=455 \mathrm{Mpc}(\mathrm{right}$ panel) respectively. Solid lines are for the nonevolution case while dash-dot lines are for the evolutionary case from Schneider et al. (2000).
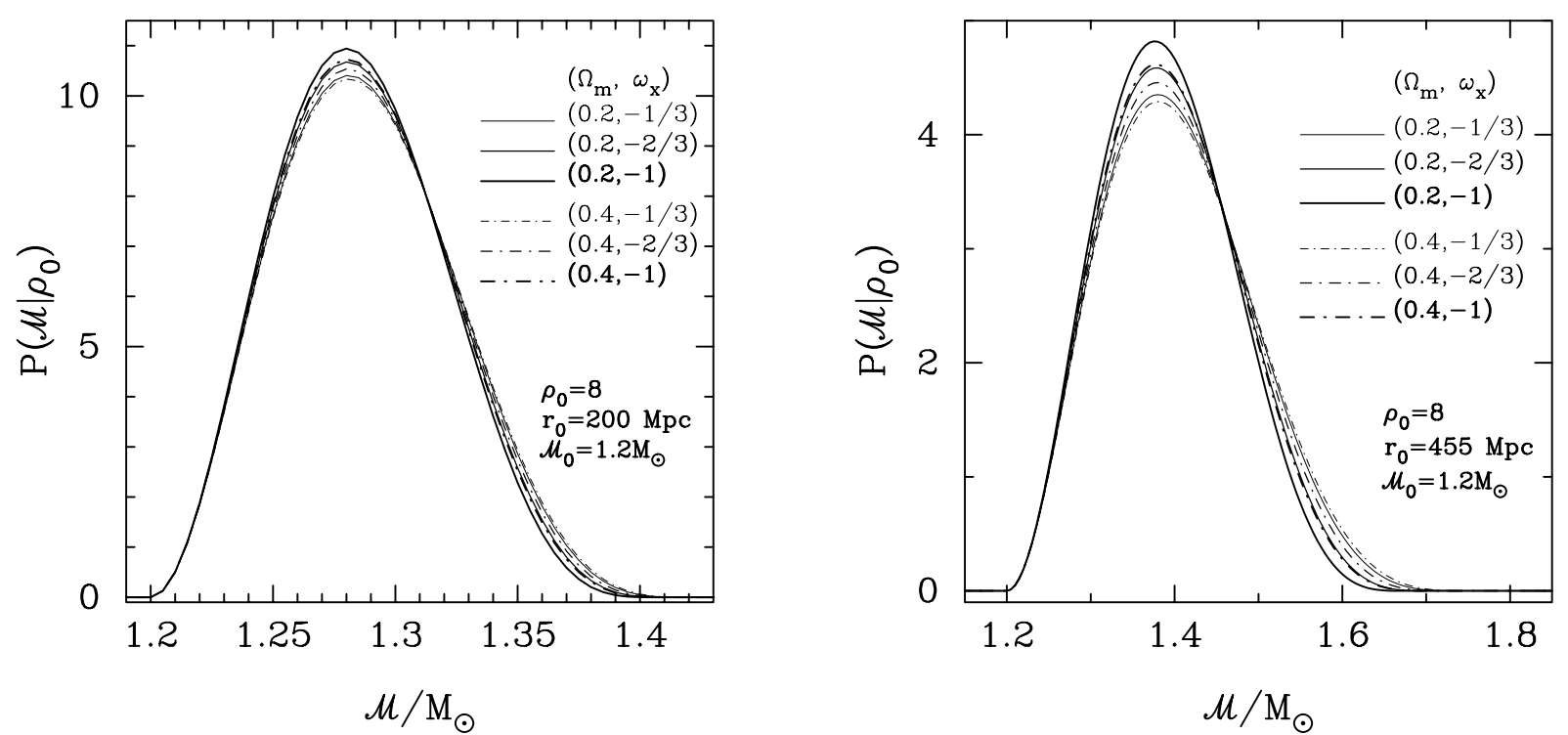

Fig. 2. The chirp mass spectrum of NS/NS binary inspirals for a flat universe with different matter density $\Omega_{m}=0.2$ (solid lines) and $\Omega_{m}=0.4$ (dash-dot lines). The detector depth is assumed to be $r_{0}=200 \mathrm{Mpc}$ (left panel) and $r_{0}=455 \mathrm{Mpc}$ (right panel) respectively.

where $i$ includes each component of matter and energy in the universe, $\omega_{i}=p_{i} / \rho_{i}$ parameterizes the effective equation of state for the $i$ th-component, $\Omega_{i} \equiv\left(8 \pi G / 3 H_{0}^{2}\right) \rho_{i 0}$, and $\Omega_{k} \equiv-k / R_{0}^{2} H_{0}^{2}$ with $H_{0}$ being the Hubble constant $\left(H_{0}=100 h \mathrm{~km} \mathrm{~s}^{-1} \mathrm{Mpc}^{-1}, 0.5 \leq h<1\right)$ and zero subscripts referring to the present epoch. Various distance measures, including $D_{\mathrm{L}}$ and $c \mathrm{~d} t / \mathrm{d} z$ that are essential to calculate the chirp mass spectrum Eq. (6) can be easily inferred from the above comoving distance (Zhu 1998; Zhu \& Cao 1999).

Figure 1 explicitly demonstrates how the chirp mass spectrum depends sensitively on the equation of state $\omega_{x}$ with both cases of NS/NS merger rate nonevolution (solid lines) and evolution (dash-dot lines). We have use two detector depths, $r_{0}=200 \mathrm{Mpc}$ for LCGT (left panel) and $455 \mathrm{Mpc}$ for LIGOII $^{1}$ (right panel), respectively. In our calculations, we use a flat universe: $\left(\Omega_{m}, \Omega_{x}\right)=(0.3,0.7)$ with a constant $\omega_{x} \in(-1,0)$, the dimensionless Hubble constant $h=0.65$ and the signal-to-noise ratio threshold $\rho_{0}=8$. As it is shown, there exists a sensitive dependence of the chirp mass spectrum of NS/NS binary inspiral on

\footnotetext{
1 http://www.ligo.caltech.edu/ ligo2/index.html http://www.ligo.caltech.edu/docs/T/T990080-00.pdf
} 
the cosmic equation-of-state. In general the smaller $\omega_{x}$, the more compressed the spectrum, and the smaller the tail at large $\mathcal{M}$. This fact may provide an independent and robust constraint on the cosmic equation of state.

\section{Discussion}

The solid lines of Fig. 1 are for the case of NS/NS merger rate nonevolution, $F(z)=1$. Unfortunately the evolution rate is probably much strong. The dash-dot lines of Fig. 1 are based on the result of Schneider et al. (2000) (Eq. (1)). As is shown, the merger rate evolution makes the spectrum less compressed and extends the tail towards large $\mathcal{M}$, which is exactly opposite to the effect for lowering $\omega_{x}$. Because uncertainties in the merger rate evolution directly lead to uncertainties in determining the cosmological equation of state, the contraints on dark energy from gravitational wave detectors could not be robust until we understand well the cosmic evolution of the NS/NS binary merger rate.

Another uncertainty of the method proposed here comes from the degenerate between the cosmic equation of state, $\omega_{x}$, and the cosmic matter density, $\Omega_{m}$. Figure 2 explicitly shows how $\Omega_{m}$ changes the spectrum. A spectrum for a flat universe with lower $\Omega_{m}$ and larger $\omega_{x}$ may overlap with the one for a flat universe with higher $\Omega_{m}$ and smaller $\omega_{x}$. One need an independent cosmological test to break down this degenerate, such as clusters of galaxies, gravitational lensing or the anisotropy of CMBR etc.

Last but not least, neutron star masses are not all identical $\left(1.37 M_{\odot}\right)$, but distribute between a lower bound (e.g. $1.29 M_{\odot}$ ) and a upper bound (e.g. $1.45 M_{\odot}$ ). This would introduce another probability function into Eqs. (3) and (4). Using a uniform distribution model, Finn (1996) shows that, the chirp mass spectrum will broaden symmetrically as the mass distribution broadens. Although the effect is different from the cosmic equation of state, the uncertainty of neutron star mass distribution will also lead the uncertainty into the constraints on dark energy from future gravitational wave events catalogue.

As is shown above, the constraints on the cosmic equation of state from the chirp mass spectrum of NS/NS merger events is promising, but it needs to appeal to statisitics, evolution and neutron star mass distributions. It is worth mentioning that, if NS/NS merger events are observed to be associated with gamma-ray bursts, one could measure the redshift of these events through the galaxies hosting the associated GRBs. Then a few NS/NS merger events would determine the cosmic equation of state as what the high- $z$ SNIa has done.

Acknowledgements. We would like to thank Prof. E. S. Phinney for many helpful suggestions, Prof. K. Kuroda for providing us the proposal materials for LCGT (Large-scale Cryogenic Gravitational Wave Telescope), Prof. D. G. Blair and Dr. D. M. Coward for helpful discussion about the cosmic evolution of NS/NS inspiral event rate. Z.-H. Zhu gratefully acknowledges support from a JSPS (the Japan Society for the Promotion of Science) fellowship.

\section{References}

Abramovici, A., et al. 1992, Science, 256, 325

Caldwell, R. R., Dave, R., \& Steinhardt, P. J. 1998, Phys. Rev. Lett., 80, 1582

Caron, B., et al. 1997, Nucl. Phys. B, Proc. Suppl., 54, 167

Carroll, S. M., Press, W. H., \& Turner, E. L. 1992, ARA\&A, 30, 499

Chernoff, D. E., \& Finn, L. S. 1993, ApJ, 411, L5

Chiba, T., Sugiyama, N., \& Nakamura, T. 1997, MNRAS, 289, L5

Cooray, A. R., \& Huterer, D. 1999, ApJ, 513, L95

Cutler, C., \& Flanagan, É. 1994, Phys. Rev. D, 49, 2658

Danzmann, K., et al. 1997, in Gravitational Wave Experiments, ed. E. Coccia, G. Pizzella, \& F. Ronga (World Scientific)

Efstathiou, G. 1999, MNRAS, 310, 842

Finn, L. S. 1996, Phys. Rev. D, 53, 2878

Finn, L. S., \& Chernoff, D. E. 1993, Phys. Rev. D, 47, 2198

Futamase, T., \& Hamana, T. 1999, Prog. Theor. Phys. Lett., 102, 1037

Garnavich, P., et al. 1998, ApJ, 509, 74

Huey, G., Wang, L., Dave, R., Caldwell, R. R., \& Steinhardt, P. J. 1999, Phys. Rev. D, 59, 063005

Kuroda, K. 1997, in Gravitational waves: Sources and Detectors, ed. I. Ciufolini, \& F. Fidecaro (World Scientific)

Kuroda, K., et al. 1999, Large-scale Cryogenic Gravitational Wave Telescope, private communication

Lima, J. A. S., \& Alcaniz, J. S. 2000a, A\&A, 357, 393

Lima, J. A. S., \& Alcaniz, J. S. 2000b, MNRAS, 317, 893

Lipunov, V. M., Postnov, K. A., Prokhorov, M. E., \& Panchenko, I. E. 1995, ApJ, 454, 593

Marković, D. 1993, Phys. Rev. D, 48, 4738

Madau, P., Della Valle, M., \& Panagia, N. 1998, MNRAS, 297, L17

Nakamura, T., \& Chiba, T. 1999, MNRAS, 306, 696

Perlmutter, S., et al. 1999, ApJ, 517, 565

Perlmutter, S., Turner, M. S., \& White, M. 1999, Phys. Rev. Lett., 83, 670

Phinney, E. S. 1991, ApJ, 380, L17

Riess, A. G., et al. 1998, AJ, 116, 1009

Schneider, R., Ferrari, V., Matarrese, S., \& Portegies Zwart, S. F. 2000, MNRAS, in press [astro-ph/0002055]

Schutz, B. F. 1986, Nature, 323, 310

Schutz, B. F. 1989, Class. Quant. Grav., 6, 1761

Spergel, D., \& Pen, U. 1997, ApJ, 491, L67

Steidel, C. C., Adelberger, K. L., Giavalisco, M., Dickinson, M., \& Pettini, M. 1999, ApJ, 519, 1

Steinhardt, P. J., et al. 1999, Phys. Rev. D, 59, 123504

Turner, M. S. 1999 [astro-ph/9904049]

Turner, M. S., \& White, M. 1997, Phys. Rev. D, 56, R4439

Tutukov, A. V., \& Yungelson, L. R. 1993, MNRAS, 260, 675

Waga, I., \& Miceli, A. P. M. R. 1999, Phys. Rev. D, 59, 103507

Wang, L., Caldwell, R. R., Ostriker, J. P., \& Steinhardt, P. J. 2000, ApJ, 530, 17

Wang, Y., \& Turner, E. L. 1997, Phys. Rev. D, 56, 724

Weinberg, S. 1989, Rev. Mod. Phys., 61, 1

Zhu, Z. H. 1998, A\&A, 338, 777

Zhu, Z. H. 2000, Int. J. Mod. Phys. D, 9, 591

Zhu, Z. H., \& Cao, L. 1999, Chin. Phys. Lett., 16, 934

Zlatev, I., Wang, L., \& Steinhardt, P. J. 1999, Phys. Rev. Lett., 82,896 